https://doi.org/10.15407/ujpe64.9.814

V.G. PANCHENKO, P.V. PORYTSKY

Institute for Nuclear Research, Nat. Acad. of Sci. of Ukraine

(47, Prosp. Nauky, Kyiv 03680, Ukraine; e-mail: vpanch@kinr.kiev.ua)

\title{
INFLUENCE OF INHOMOGENEITY ON TRANSFORMATION AND RADIATION PROCESSES IN PLASMA WITH UPPER HYBRID PUMP
}

\begin{abstract}
On the basis of the kinetic theory of fluctuations, the processes of the longitudinal Langmuir wave transformation into a transverse electromagnetic wave in the turbulent inhomogeneous plasma have been studied. The plasma turbulence is assumed to arise owing to the parametric decay of the upper hybrid pump wave into a daughter wave and electron-drift oscillations. The transformation coefficient under the parametric instability saturation conditions is determined. The intensity of the electromagnetic radiation emission from the plasma is calculated, and its dependence on the plasma and pump wave parameters is found.
\end{abstract}

Keywords: kinetic theory of fluctuations, turbulent plasma, upper hybrid pump, transformation, plasma radiation.

\section{Introduction}

The study of the scattering and transformation processes is known to be challenging for both the research of the fundamental plasma properties and the solution of various practical problems dealing with a plasma, e.g., the plasma diagnostics, the study of the mechanisms of wave transformation, and the measurement of the efficiency of the RF power dissipation in a plasma. Rather important seems to be the study of the role of fluctuations in those processes. It is evident that the intensity of the scattering or transformation will be quite substantial in a non-equilibrium plasma, if the magnitude of fluctuations considerably exceeds that of the thermal noise.

The plasma non-equilibrium state can be associated, e.g., with the development of parametric instabilities induced by the influence of the pumping on a plasma. Note that the study of various nonlinear processes in a "plasma-with-pump" system is of permanent interest, which is evidenced by recent researches dealing with a laboratory plasma [1,2], a plasma of fusion devices [3, 4], and a quantum plasma [5].

The theory of the transformation and scattering of electromagnetic waves in a plasma was developed in works [6-9] and described in works $[10,11]$ in detail. Various possibilities of using the scattering and

(C) V.G. PANCHENKO, P.V. PORYTSKY, 2019 transformation for the diagnostics of laboratory and space plasmas were analyzed in work [12].

The kinetic theory of fluctuations in a plasma has been developed in works [13-15]. The influence of the electromagnetic radiation of various types (a dc electric field, an oscillating electric field with a finite amplitude, high-power radiation, a pump wave) on fluctuations in a magnetoactive plasma was analyzed in works $[11,16-18]$. The theory of fluctuations in a magnetized plasma in the presence of parametric instabilities of pump waves in various frequency ranges was developed further in works [19-21].

Various transformation processes in a magnetized turbulent plasma with low hybrid pumps were considered in works $[22,23]$. The transformation in a turbulent plasma with upper hybrid pump was studied in work [24]. The results obtained in the cited works made it possible to explain the mechanism of electromagnetic radiation emission from a plasma, in particular, the spontaneous radiation emission from Sun's corona at the double Langmuir frequency.

In this work, on the basis of the kinetic theory of fluctuations, the processes of transformation of the longitudinal Langmuir wave into an electromagnetic wave in a magnetically inhomogeneous and parametrically unstable plasma have been studied. The transformation coefficient is obtained in the case where electron-drift oscillations are parametrically excited

ISSN 2071-0194. Ukr. J. Phys. 2019. Vol. 64, No. 9 
by the upper hybrid pump. The intensity of the electromagnetic radiation emission from a plasma is calculated, and its dependence on the plasma and pump wave parameters is determined.

It should be noted that, in our previous works devoted to the study of the processes of scattering and transformation, the case of uniform plasma was mainly considered. Under real laboratory conditions, however, the plasma is always inhomogeneous. Therefore, the application of the inhomogeneous plasma model is very important, because it allows a number of problems associated with the parametric excitation of low-frequency oscillations in a plasma - in particular, various types of drift waves appearing owing to the existence of the density and temperature gradients in a plasma - to be considered. An important role of parametric instabilities in the frequency range of the upper hybrid resonance was marked in works $[25,26]$, in which the anomalous absorption of RF power and the wave scattering processes in a turbulent plasma were analyzed.

\section{Formulation of the Problem}

A dominant contribution to the differential crosssection of transformation, $d \Sigma_{l \rightarrow t}$, is given by electron density fluctuations. At the same time, the interaction between the incident Langmuir wave and the fluctuations of electric and magnetic fields, as well as the thermal velocity of electrons, can be neglected. Therefore, the differential cross-section $d \Sigma_{l \rightarrow t}$ can be written as follows $[11,22]$ :

$d \Sigma_{l \rightarrow t}=\frac{1}{2 \pi}\left(\frac{e^{2}}{m c^{2}}\right)^{2} \frac{\omega^{\prime 4}}{\omega_{p e}^{4}} N\left\langle\delta n_{e}^{2}\right\rangle_{\mathbf{q}, \Delta \omega} d \omega^{\prime \prime} d \mathrm{O}$,

where $\Delta \omega=\omega^{\prime}-\omega^{\prime \prime} ; \mathbf{q}=\mathbf{k}^{\prime}-\mathbf{k}^{\prime \prime} ;\left(\omega^{\prime}, \mathbf{k}^{\prime}\right)$ and $\left(\omega^{\prime \prime}, \mathbf{k}^{\prime \prime}\right)$ are the frequencies and wave vectors of the incident and transformed waves, respectively; $\mathrm{O}$ is the solid angle; and $\left\langle\delta n_{e}^{2}\right\rangle_{\mathbf{q}, \Delta \omega}$ is the correlator of electron density fluctuations at the combinational frequency $\Delta \omega$ (the formula for this parameter can be found, e.g., in work [22]). The factor $N$ in formula (1) is determined by the known expression $[11,24]$ and depends on the direction of the incident and transformed waves with respect to the magnetic field. Attention should also be paid to that the frequencies and wave vectors of the incident and transformed waves are mutually related by the energy and momentum conservation laws,

$\omega^{\prime \prime}=\omega^{\prime}+\Delta \omega, \quad \mathbf{k}^{\prime \prime}=\mathbf{k}^{\prime}+\mathbf{q}$.
Let us consider the electron-ion plasma embedded into an external magnetic field. The plasma is affected by an HF pump field, which is directed perpendicularly to the magnetic field. The pump field will be considered in the dipole approximation, $\mathbf{E}_{0}(t)=$ $=E_{0} \mathbf{y} \cos \omega_{0} t$, with the pump frequency being in the upper hybrid frequency range, i.e., $\omega_{0} \propto \omega_{\mathrm{UH}}$, where

$\omega_{\mathrm{UH}}=\Omega_{e}\left(1+\frac{\omega_{p e}^{2} \sin ^{2} \theta}{2 \Omega_{e}^{2}}\right)$.

Note that expression (3) is valid in the case of highly magnetized plasma, when $\omega_{p e} \ll \Omega_{e}$. Let us also assume that the decay decrement of the upper hybrid wave equals $\gamma_{\mathrm{UH}} \approx \nu_{e i}$.

The plasma inhomogeneity is characterized by an exponential gradient of the density, when the corresponding distribution function $f_{0 e, i}$ is proportional to $\exp (\alpha y)$ with the plasma inhomogeneity parameter $\alpha=\left(1 / n_{0}\right)\left(d n_{0} / d y\right)$. As was shown in work [27], the ratio between the pressure and magnetic field inhomogeneities is of an order of $L_{n} / L_{B} \propto \beta$, where $L_{n}$ and $L_{B}$ are the characteristic scales of changes for the plasma density and the magnetic field, respectively, and $\beta$ is the ratio between the plasma and magnetic pressures. The condition $\beta \ll 1$ is well obeyed in most cases of a laboratory plasma, as well as for ionospheric plasma. Therefore, the inhomogeneity of the magnetic field will be neglected in comparison with the inhomogeneity of the plasma density. Temperature gradients will also be neglected.

It is known [28] that, in a magnetoactive inhomogeneous plasma, there can exist electron-drift oscillations, whose frequency and damping decrement are determined by the formulas

$\omega_{D} \approx-\frac{k_{\perp} \alpha T_{e}}{m_{e} \Omega_{e}}$

and

$\gamma_{D}=-\left(\frac{\pi}{2}\right)^{1 / 2} \omega_{D}\left[\frac{-\omega_{D} k_{x}^{2} \rho_{i}^{2}\left(1+T_{e} / T_{i}\right)}{2 k_{\|} v_{T e}}+\right.$

$\left.+\frac{T_{e}}{T_{i}} \frac{\omega_{D}\left(1+T_{i} / T_{e}\right)}{2 k_{\|} v_{T i}} \exp \left(-\frac{\omega_{D e}^{2}}{2 k_{\|}^{2} v_{\|}^{2}}\right)\right]$,

respectively. From Eq. (5), one can see that the interaction of the intrinsic plasma waves with electrons is a destabilizing factor, i.e. the excitation of oscillations takes place, which is caused by the convection of resonance electrons, since the ion damping is 
exponentially low. Note, however, that the Landau ion damping can be significant for a plasma in small laboratory installations, where $k_{||}$acquires large values. Furthermore, the damping in installations with a magnetic field shear is a result of the ion convection into the region, where $k_{\|}$is large, whereas small $k_{\|}$-values occur only in narrow plasma layers [20].

Below, we will consider the case where the drift oscillations fade, i.e. the contribution of the second (ion) term in formula (5) is crucial.

\section{Transformation of Langmuir Wave in Turbulent Plasma}

Let us consider the transformation process under conditions of parametric instability, when the pump wave decays into a daughter upper hybrid wave and electron-drift oscillations:

$\omega_{0}=\omega_{\mathrm{UH}}+\omega_{D}$.

Note that the pump frequency $\omega_{0}$ has to slightly exceed the upper hybrid frequency $\omega_{\mathrm{UH}}$, because the relation $\omega_{D} \ll\left(\omega_{0}, \omega_{\mathrm{UH}}\right)$ is obeyed.

Provided that the decay condition (6) is satisfied, it is easy to obtain an expression for the threshold field strength with respect to this parametric instability,

$E_{*}^{2} \approx \frac{8 \omega_{0}^{2} B_{0}^{2}}{k^{2} c^{2}}\left(q r_{D e}\right)^{2} \frac{\omega_{p e}^{2}}{\Omega_{e}^{2}} \frac{\gamma_{\mathrm{UH}}}{\omega_{\mathrm{UH}}} \frac{\gamma_{D}}{\omega_{D}}$.

By integrating expression (1) over $\omega^{\prime \prime}$ and substituting values for the electron density correlator, the transformation cross-section can be written in the form $[18,19]$

$\frac{d \Sigma_{l \rightarrow t}}{d \mathrm{O}}=\frac{d \Sigma_{+}}{d \mathrm{O}}+\frac{d \Sigma_{-}}{d \mathrm{O}}$,

where

$$
\begin{aligned}
& \frac{d \Sigma_{ \pm}}{d \mathrm{O}} \approx \frac{3}{16 \pi^{2}}\left(\frac{e^{2}}{m c^{2}}\right)^{2} \frac{\omega^{4}}{\omega_{p e}^{4}} N \frac{\pi}{\operatorname{Im} \varepsilon_{E} \times \frac{\partial \operatorname{Re} \varepsilon_{E}}{\partial \Delta \omega}} \times \\
& \times\left[\left|1+\chi_{i}^{0}+\frac{\mu^{2}}{4} \chi_{e}^{0} \chi_{i}^{0} \frac{1}{\varepsilon_{-1}}\right|^{2}\left\langle\delta n_{e}^{2}\right\rangle \stackrel{\Delta \omega, \mathbf{q}}{0}+^{0}\right. \\
& +\left|\chi_{e}^{0}\right|^{2}\left\langle\delta n_{i}^{2}\right\rangle_{\Delta \omega, \mathbf{q}}^{0}+ \\
& \left.+\frac{\mu^{2}}{4}\left|\chi_{e}^{0}\right|^{2}\left|\chi_{i}^{0}\right|^{2} \frac{\left\langle\delta \mathbf{E}^{2}\right\rangle{ }_{\Delta \omega \mp \omega_{0}, \mathbf{q}}^{0}}{\left|\varepsilon_{\mp 1}\right|^{2}}\right] .
\end{aligned}
$$

Here, $\Delta \omega=\omega_{\mathrm{UH}}$ for the upper hybrid wave and $\Delta \omega=$ $=\omega_{D}$ for the drift one, and $\chi_{\alpha}^{0}(\alpha=e, i)$ is the linear susceptibility of a plasma. Note that the expression for the nonlinear dielectric constant $\varepsilon_{E}$ is given in works $[19,20]$. The parameter

$\mu=\frac{k_{\perp} E_{0} c}{\omega_{0} B_{0}} \ll 1$.

This criterion can be considered as a restriction imposed from above on the pump wave field amplitude. At the same time, there exists an analogous restriction from below, since we consider the turbulent plasma mode in the parametric instability region above the threshold, i.e. when $E_{0}>E_{*}$.

In formula (9), the quantities $\left\langle\delta n_{\alpha}^{2}\right\rangle^{0}$ and $\left\langle\delta \mathbf{E}^{2}\right\rangle^{0}$ are the known spectral correlation functions for the plasma density and electric field fluctuations, respectively, in the case of non-interacting particles in a magnetized plasma and in the absence of an electric field $[15,18,25]$. In the above-threshold region of parametric instability, the plasma transits into a mode with developed fluctuations. In order to determine the saturation level for fluctuations, we use the stabilization mechanism for the instability invoked by the scattering of charged particles on turbulent fluctuations of the electric field.

To characterize this scattering, let us introduce the effective collision frequency $\nu_{\text {eff }}$ and assume that $\nu_{\text {eff }}>\left(\gamma_{\mathrm{UH}}, \gamma_{D}\right)$. In this case, when calculating the correlators for the density and electric fluctuations regarded as characteristic plasma waves, we will consider the quantities $\tilde{\omega}=\omega+i \nu_{\text {eff }}$ and use the reevaluated threshold field magnitude $\tilde{E}_{*}^{2} \approx \frac{E_{*}^{2} \nu_{\text {eff }}^{2}}{\gamma_{U} \gamma_{D}}$, where $\nu_{\text {eff }}=\frac{E_{0}^{2}}{E_{*}^{2}}\left(\gamma_{\mathrm{UH}} \gamma_{D}\right)^{1 / 2}$. This mechanism of instability saturation, which considers the additional wave damping, was described in works $[18,20]$ in detail. It should be noted that, in the above-threshold region of parametric instability and in the mode of turbulent fluctuations, the effective collision frequency exceeds the damping decrement of the upper hybrid and drift waves, but remains undoubtedly lower than the actual frequencies of the waves that are decay products [see Eq. (6)].

Let us discuss another issue. In this work, we do not give expressions for the correlators of the density and electric field fluctuations obtained in the previous works for a uniform plasma. It is known $[11,14]$ that the spectral densities of the field and density 
fluctuations are expressed in terms of the correlators of non-interacting particles, which depend, in turn, on the correlator of source fluctuations. In an inhomogeneous plasma, the latter depends not only on the difference $\mathbf{r}-\mathbf{r}^{\prime}$, but also on the vectors $\mathbf{r}$ and $\mathbf{r}^{\prime}$ separately. For a weakly inhomogeneous plasma, the dependence of the source fluctuation correlator on $\mathbf{r}-\mathbf{r}^{\prime}$ is more abrupt than the dependence on the coordinate $\mathbf{r}$, since the former is associated with the wavelength of drift oscillations $\lambda=2 \pi / k_{\perp}$, whereas the latter with the characteristic scale of the plasma inhomogeneity $L=1 / \alpha$. Therefore, the condition of weak inhomogeneity, $\lambda \ll L$, is satisfied.

\section{Results of Calculations}

As was done in the previous works $[23,26]$, lowfrequency electron-drift oscillations are assumed to make a dominant contribution to the electron density correlator $\left\langle\delta n_{e}^{2}\right\rangle_{\mathbf{q}, \Delta \omega}$ and, thus, to the transformation cross-section (9). In this case, we may set

$$
\begin{aligned}
& \left|\chi_{e}^{0}\right| \approx \frac{1}{q^{2} r_{D e}^{2}}, \\
& \left|\chi_{i}^{0}\right| \approx \frac{1}{q^{2} r_{D i}^{2}}\left(1+\frac{k_{\|}^{2} v_{T i}^{2}}{\omega^{2}}\right), \\
& \operatorname{Im} \varepsilon_{E} \approx \frac{1}{q^{2} r_{D e}^{2}} \frac{\gamma_{D}}{\omega_{D}}\left(1-\frac{E_{0}^{2}}{\tilde{E}_{*}^{2}}\right), \\
& \frac{\partial \operatorname{Re} \varepsilon_{E}}{\partial \omega_{D}} \approx \frac{1}{q^{2} r_{D e}^{2}} \frac{1}{\omega_{D}}, \\
& \left\langle\delta \mathbf{E}^{2}\right\rangle_{\Delta \omega \mp \omega_{0}, \mathbf{q}}^{0} \approx 16 \pi T_{e} \frac{\gamma_{\mathrm{UH}}}{\omega_{p e}^{2}}, \\
& \left\langle\delta n_{i}^{2}\right\rangle_{\omega_{D}, \mathbf{q}}^{0}=\frac{q^{2}}{16 \pi e^{2}} \frac{8 \pi}{\omega_{D}} T_{i} \frac{1}{q^{2} r_{D e}^{2}} \frac{\gamma_{D}}{\omega_{D}} .
\end{aligned}
$$

Here, when calculating the electric field and density correlators for non-interacting particles, we used the fluctuation-dissipative theorem. Keeping only the resonance terms in Eq. (9) and making allowance for formulas (10), the following expression for the transformation cross-section is obtained:

$$
\begin{aligned}
& \frac{d \Sigma_{ \pm}}{d \mathrm{O}} \approx \frac{1}{4 \pi}\left(\frac{e^{2}}{m c^{2}}\right)^{2} \frac{\omega^{4}}{\omega_{p e}^{4}} N \frac{T_{i}}{T_{e}} n_{e} \times \\
& \times\left(1+\frac{\mu^{2}}{8} \frac{1}{q^{2} r_{D e}^{2}} \frac{T_{e}}{T_{i}} \frac{\Omega_{e}^{2} \omega_{D}^{2}}{\omega_{\mathrm{UH}}^{2} \gamma_{\mathrm{UH}} \gamma_{D}}\right) .
\end{aligned}
$$

Let us evaluate this expression. For the typical parameters of a hot plasma $T_{e}=5 \mathrm{keV}, B_{0}=50 \mathrm{kGs}$, $n_{e}=10^{14} \mathrm{~cm}^{-3}$, and the pump field with an energy flux density of $50 \mathrm{~kW} / \mathrm{cm}^{2}$ [20], which is used for the heating of such plasma, we obtain $\mu \propto 10^{-1}$. We may also put $q r_{D e} \approx 10^{-1}$ and $\omega_{D} / \gamma_{D} \geq 10[29,30]$. The corresponding calculations show that the main contribution to the transformation coefficient is given by the second term in the parentheses on the righthand side of expression (11). This term depends on the pump field amplitude and can exceed 1 (this value corresponds to the wave transformation induced by the thermal noise) by several orders of magnitude. Note also that, in this case, the transformation cross-section substantially depends on the plasma density gradient $\left(d \Sigma_{l \rightarrow t} \propto \alpha\right)$.

The transformation of longitudinal waves into electromagnetic ones also comprises a point of interest as one of the possible mechanisms governing the energy emission from a plasma by means of electromagnetic waves. Really, there are always stochastic Langmuir waves in a plasma, whose amplitudes are determined by the electron temperature. By interacting with turbulent drift oscillations, the Langmuir waves can transform into electromagnetic ones, which are emitted from the plasma afterward. The corresponding radiation intensity is related to the transformation coefficient by the relation [11]

$I \approx V \iint d \Sigma_{l \rightarrow t} S_{\omega^{\prime}, \mathbf{k}^{\prime}}^{\prime} \frac{d^{3} k^{\prime}}{(2 \pi)^{3}} \frac{d \omega^{\prime}}{2 \pi}$

where $V$ is the plasma volume, and $S_{\omega^{\prime}, \mathbf{k}^{\prime}}^{\prime}$ is the energy flux density of fluctuating Langmuir waves, which is determined by the expression

$S_{\omega^{\prime}, \mathbf{k}^{\prime}}^{\prime} \approx \frac{v_{g r}}{16} \frac{\partial}{\partial \omega^{\prime}}\left(\varepsilon^{l} \omega^{\prime}\right)\left\langle\delta \mathbf{E}^{2}\right\rangle \omega_{\omega^{\prime}, \mathbf{k}^{\prime}}^{0}$

In formula (13), $v_{g r}=3 \omega_{p e} r_{D e}^{2} k^{\prime}$ is the group velocity of Langmuir waves, and

$\varepsilon^{l}=1-\frac{\omega_{p e}^{2}}{\omega^{2}-\Omega_{e}^{2}} \sin ^{2} \theta-\frac{\omega_{p e}^{2}}{\omega^{2}} \cos ^{2} \theta$

is the dielectric constant for longitudinal Langmuir waves.

We may take

$\frac{\partial}{\partial \omega^{\prime}}\left(\varepsilon^{l} \omega^{\prime}\right)=1+\frac{2 \omega_{p e}^{2}}{\omega^{\prime 2}} \approx 3$

at $\omega^{\prime} \approx \omega_{p e}$ and assume that the inequality $k^{\prime \prime} \ll k^{\prime} \propto$ $\propto q$ is satisfied for the process $l \rightarrow t$. Substituting 
only the dominant (field) term from Eq. (11) into Eq. (12), taking into account that $\int\left\langle\delta \mathbf{E}^{2}\right\rangle{ }_{\omega^{\prime}, \mathbf{k}^{\prime}}^{0} d \omega^{\prime} \propto$ $\propto T_{e}$, and integrating over $k^{\prime}$ in Eq. (12), we obtain a final expression for the intensity of electromagnetic radiation emission from a turbulent plasma:

$$
\begin{aligned}
& I \approx V\left(\frac{e^{2}}{m c^{2}}\right)^{2} N T_{i} n_{e} \omega_{p e}\left(k^{\prime} r_{D e}\right)^{2} \times \\
& \times\left(1+\frac{\mu^{2}}{8} \frac{1}{q^{2} r_{D e}^{2}} \frac{T_{e}}{T_{i}} \frac{\Omega_{e}^{2} \omega_{D}^{2}}{\omega_{\mathrm{UH}}^{2} \gamma_{\mathrm{UH}} \gamma_{D}}\right) .
\end{aligned}
$$

The corresponding numerical calculations show that, in the case of typical plasma parameters that were used above to estimate expression (11), the value of the electromagnetic radiation intensity given by formula (14) is much larger than the corresponding value obtained for a magnetized plasma in the absence of turbulence.

\section{Conclusions}

In this work, the processes of transformation of the longitudinal wave into an electromagnetic one at turbulent fluctuations in a magnetoactive plasma in the above-threshold region of parametric instability have been studied. The turbulent instability arises when electron-drift oscillations are parametrically excited by the upper hybrid pump wave. It is shown that the main contribution to the transformation coefficient and the intensity of radiation emission from a plasma is made by the terms that depend on the frequency and amplitude of the pump field. The dependences of the indicated quantities on the density gradient [Eqs. (11) and (14)] makes it possible to elucidate the influence of the plasma inhomogeneity on the processes of transformation in and radiation emission from a turbulent plasma. In addition, by changing the pump parameters, it is possible to control the electromagnetic radiation emission from the plasma medium, which can be important for the diagnostics of laboratory and space plasmas.

1. Q. Chen, Z. Wu, L. Johnson, D. Gordon, P. Sprangle, S. Suckewer. Pulse splitting of stimulated Raman backscattering with a chirped pump. Phys. Plasmas 24, 052115 (2017).

2. E.Z. Gusakov, A.Yu. Popov, P.V. Tretinnikov. Kinetic theory of electrostatic waves nonlinear coupling in the magnetized plasma in presence of extraordinary pump wave. Plasma Phys. Control. Fusion 61, 085008 (2019).
3. Y. Oda, R. Ikeda, K. Kajiwara, T. Kobayashi, K. Hayashi, K. Takahashi, S. Moriyama, K. Sakamoto, T. Eguchi, Y. Kawakami et al.. Development of the first ITER gyrotron in QST. Nucl. Fusion 59, 086014 (2019).

4. E.Z. Gusakov, A.Yu. Popov. Saturation of the low-threshold parametric decay of the O-mode pump in the fundamental harmonic ECRH experiment. Phys. Plasmas 25, 082117 (2018).

5. F. Sayed, S.V. Vladimirov, Yu. Tyshetskiy, O. Ishihara. Modulational and filamentational instabilities of a monochromatic Langmuir pump wave in quantum plasmas. Phys. Plasmas 25, 052115 (2015).

6. A.I. Akhiezer, I.G. Prohoda, A.G. Sitenko. Scattering of electromagnetic waves in a plasma. J. Exper. Theor. Phys. 6, 576 (1958)

7. J.P. Dougherty, D.T. Farley. A theory of incoherent scattering of radio waves by a plasma. Proc. Roy. Soc. A $\mathbf{2 5 9}$, 79 (1961).

8. M.N. Rosenbluth, N. Rostoker. Scattering of electromagnetic waves by a nonequilibrium plasma. Phys. Fluids 5, 776 (1962).

9. E.E. Salpeter. Electron density fluctuations in a plasma. Phys. Rev. 120, 1528 (1960).

10. J. Bekefi. Radiation Processes in Plasmas (Wiley, 1966).

11. A.I. Akhiezer, I.A. Akhiezer, R.V. Polovin, A.G. Sitenko, K.N. Stepanov Plasma Electrodynamics (Pergamon Press, 1978) [ISBN: 978-0-08-017783-0].

12. V.E. Golant. High-Frequency Wave Effects on the Shaping and Heating of Plasma in a High-Frequency Magnetic Field (Nauka, 1968) (in Russian).

13. V.Yu. Bychenkov, V.P. Silin. A kinetic equation for a plasma in a high-frequency field. J. Exper. Theor. Phys. 75, 40 (1975).

14. A.G. Sitenko. Fluctuations and Non-Linear Wave Interaction in Plasmas (Pergamon, 1982). [ISBN: 978-0-08025051-9].

15. V.N. Pavlenko, V.G Panchenko, S.M. Revenchuk. Anomalous absorption of electromagnetic wave energy near the lower hybrid resonance. Plasma Phys. Contr.Fusion 26, 1221 (1984).

16. Yu.L. Klimontovich. Kinetic Theory of Nonideal Gases and Nonideal Plasmas (Pergamon, 1982) [ISBN: 978-0-08021671-3].

17. V.P. Silin. Parametric Effects of High-Power Radiation on Plasma (Nauka, 1973) (in Russian).

18. V.N. Pavlenko, V.G Panchenko, S.M. Revenchuk. Turbulent conductivity of a magnetoactive plasma. J. Exper. Theor. Phys. 64, 50 (1986).

19. H. Wilhelmsson, V.N. Pavlenko, V.G Panchenko. Electromagnetic wave scattering by a turbulent plasma in the presence of a lower hybrid pump. Phys. Scripta 43, 323 (1991).

20. V.N. Pavlenko, V.G Panchenko. Parametric absorption of lower hybrid waves in a nonuniform magnetized plasma. Plasma Phys. Rep. 25, 288 (1999).

ISSN 2071-0194. Ukr. J. Phys. 2019. Vol. 64, No. 9 
21. L. Stenflo. Nonlinear propagation of electromagnetic waves in magnetized electron-positron plasma. Astrophys. Space Sci. 117, 303 (1985).

22. V.N. Pavlenko, V.G Panchenko. Transformation of electromagnetic waves in turbulent magnetized plasma. Phys. Scripta 81, 502 (2010)

23. V.N. Pavlenko, V.G Panchenko. Radiation processes in turbulent plasma with ionic temperature anisotropy. Phys. Scripta 87, 055503 (2013).

24. V.N. Pavlenko, V.G Panchenko. Double Langmuir frequency radiation due to transformation processes in turbulent plasma. J. Plasma Phys. 81, 0022377814000932 (2015).

25. V.N. Pavlenko, V.G Panchenko, L. Stenflo, H. Wilhelmsson. Absorption of upper hybrid pump energy in a turbulent magnetized plasma. Phys. Scripta 45, 616 (1992).

26. V.N. Pavlenko, V.G Panchenko, S.F. Nazarenko. Nonlinear scattering processes in magnetized plasmas with highfrequency pumps. Phys. Scripta 82, 109 (1999).

27. V.L. Ginzburg, A.A. Rukhadze Waves in Magnetoactive Plasma (Nauka, 1975) (in Russian).

28. A.B. Mikhailovskii. Theory of Plasma Instabilities. Vol 2: Instabilities of an Inhomogeneous Plasma (Springer, 1974) [ISBN: 978-1-4899-4787-1].

29. M. Porkolab. Parametric instabilities due to lower-hybrid radio frequency heating of tokamak plasmas. Phys. Fluids 20, 2058 (1977).
30. L.V. Krupnova, V.T. Tikhonchuk. Parametric absorption of electromagnetic radiation in a magnetized plasma. J. Exper. Theor. Phys. 79, 917 (1979).

Received 07.06.19.

Translated from Ukrainian by O.I. Voitenko

В.Г. Панченко, П.В. Порицъкий

ВПЛИВ НЕОДНОРІДНОСТІ

НА ПРОЦЕСИ ТРАНСФОРМАЦІЇ

І ВИПРОМІНЮВАННЯ В ТУРБУЛЕНТНІЙ

ПЛАЗМІ З ВЕРХНЬОГІБРИДНОЮ НАКАЧКОЮ

$\mathrm{P}$ е $з$ ю м е

На основі кінетичної теорії флуктуацій вивчені процеси трансформації поздовжньої ленгмюрівської хвилі в електромагнітну хвилю в турбулентній неоднорідній плазмі. Турбулентність плазми зумовлена параметричним розпадом верхньогібридної хвилі накачки на дочірню та електронно-дрейфові коливання. Отримано коефіціент трансформації в умовах насичення параметричної нестійкості. Обчислена інтенсивність електромагнітного випромінювання з плазми та знайдена її залежність від параметрів плазми і хвилі накачки. 